\title{
Cyclic vomiting syndrome: the nervous system has the guts
}

\author{
Ryuji Sakakibara ${ }^{1}$
}

Received: 14 February 2018 / Accepted: 16 February 2018 / Published online: 22 February 2018

c) Springer-Verlag GmbH Germany, part of Springer Nature 2018

In this issue of Clinical Autonomic Research, Bhandari and colleagues publish a comprehensive state-of-the-art review on cyclic vomiting syndrome [1]. As described in this article, cyclic vomiting syndrome is a difficult, presumably neural as well as psychological condition. The neural etiology appears to be the underlying pathophysiology in cannabis users, whereas the psychological background is more evident in non-cannabis users.

Vomiting is a relatively common occurrence in the adult and pediatric populations, with up to $6 \%$ of pediatric patients complaining of functional nausea and vomiting [2]. When these patients present to clinic, practitioners typically perform a work-up to rule out systemic disorders by blood tests, upper endoscopy, abdominal imaging, or other diagnostic modalities focused on the gastrointestinal tract itself. What most general practitioners and gastroenterologists perhaps forget is that the gastrointestinal tract is intimately controlled by the central and peripheral nervous systems. Indeed, the autonomic nervous system is involved in the initiation and expression of emesis, in response to a number of stimuli, including endogenous cannabinoids binding to cannabinoid receptors located in central and peripheral pathways [3] (Fig. 1).

The link between the endocannabinoid system and the autonomic nervous system is increasingly documented. A recently published article in Clinical Autonomic Research reviewed the endocannabinoid mechanisms involved in autonomic cardiovascular control [8]. In this regard, several gastrointestinal disorders featuring prominent nausea and vomiting, such as cyclic vomiting syndrome, are associated with clinical manifestations of autonomic dysfunction and objective abnormalities on autonomic function testing. Conversely, autonomic dysfunction caused by neurodegeneration (such as Parkinson disease and other synucleinopathies), genetic mutations (e.g., familial dysautonomia), or focal brain lesions in the dorsal vagal complex (Fig. 1) [7] can present with disabling nausea and vomiting. In the case of familial dysautonomia, the mechanism of nausea is related to massive dopamine release [5]. Of note, in patients with Parkinson disease treatment with L-dopa also triggers nausea, which can be relieved by blocking the peripheral conversion of L-dopa to dopamine with carbidopa, a dopadecarboxylase inhibitor. This is why L-dopa is commercialized in combination with carbidopa [4]. Carbidopa (without L-dopa) also relieves hyperdopaminergic-vomiting crisis in patients with familial dysautonomia [6]. Whether dopamine pathways are also involved in cyclic vomiting syndrome is unknown, but the hypothesis is tempting.

In the meantime, as described by Bhandari and colleagues [1], available therapies acting on dopaminergic and serotoninergic receptors in the nervous system are effective at attenuating nausea and vomiting. This is also the case with the most extensively studied etiology of severe emesis, i.e., chemotherapy-induced nausea and vomiting, which is also preventable and treatable by agents targeting neurokinin, dopamine, serotonin, and endocannabinoid receptors located in the peripheral and central nervous systems. These few examples provide insights of potentially effective therapies

Ryuji Sakakibara

sakakibara@ sakura.med.toho-u.ac.jp

1 Neurology Division, Department of Internal Medicine, Sakura Medical Center, Toho University, 564-1 Shimoshizu, Sakura 285-8741, Japan 
Fig. 1 Neural circuits involved in emesis. a Afferent vagal stimulation in decerebrated paralyzed dogs leads to emesis by progressive activation of:

(1) neurons of the nucleus of the solitary tract (mNTS);

(2) neurons of the prodromal sign center near the pars semi-compacta of the nucleus ambiguus (scAMB); (3) neurons of the central pattern generator near the pars compacta of the nucleus ambiguous (cAMB);

(4) respiratory premotor neurons of the diaphragm (Ip) and abdominal muscle (Ep) premotor neurons in the caudal medulla; and v) motor neurons of the diaphragm (Im) and abdominal muscles (Em). $C P G$ central pattern generator for vomiting. $\mathbf{b} \mathrm{T}_{2}$-weighted sagittal image showing a focal medullary lesion in a 23-year-old woman diagnosed with neurolupus presenting with isolated vomiting. $\mathbf{c}$ and $\mathbf{d ~ T}_{2}$-weighted axial images showing a high-signal-intensity lesion in the right dorsolateral medulla (figures are reversed to match the illustration on panel E). e Figure illustrating the area of the lesion (hatched area) in the axial MRI and the location of the inferior vestibular nucleus and the dorsal vagal complex, corresponding to the vomiting pattern generator, which includes the area postrema, i.e., the chemo-sensitive trigger zone. $D V C$ dorsal vagal complex, $I V$ inferior vestibular nucleus, $A P$ area postrema, med medial, NTS nucleus tractus solitarius, gel subnucleus gelatinosus, cen subnucleus centralis, int intermediate subnucleus, is interstitial subnucleus, $T S$ tractus solitarius, $v l$ ventrolateral subnucleus of NTS, DMN dorsal motor nucleus, $X 11$ hypoglossal nucleus, V4 fourth ventricle

\section{A Neuronal circuit \\ Patterns of neuronal firings}
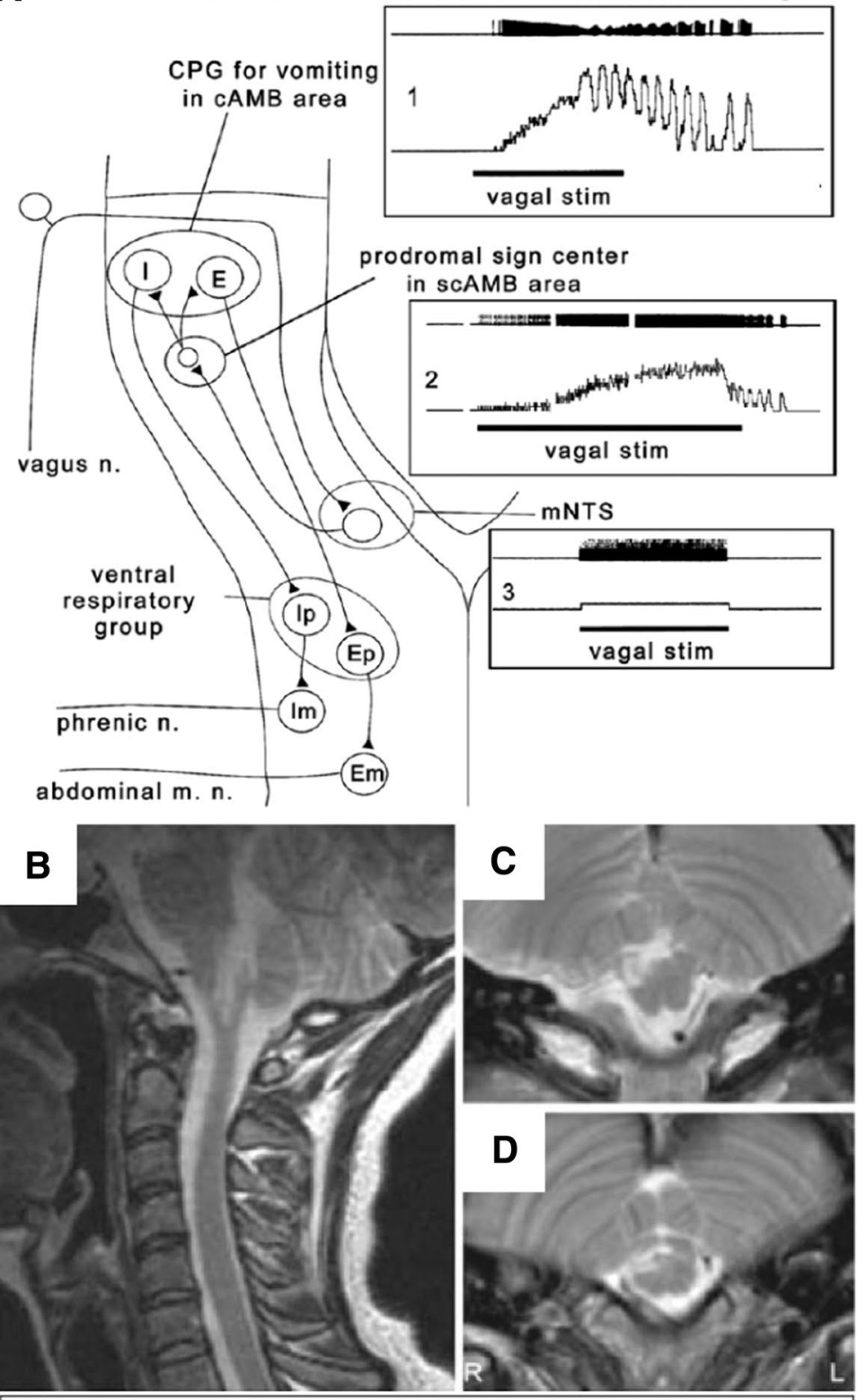

E

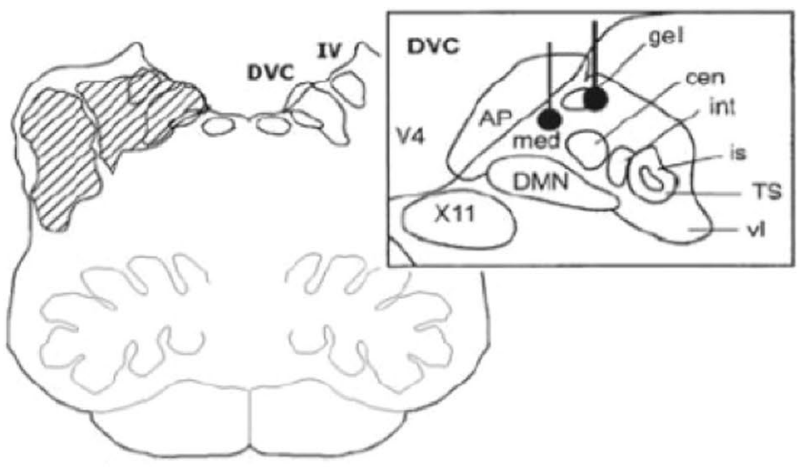


for patients with cyclic vomiting syndrome and highlight how, in the case of vomiting nausea, the nervous system has the guts.

Funding None.

\section{Compliance with ethical standards}

Conflict of interest The author declares that they have no conflict of interest.

\section{References}

1. Bhandari S, Jha P, Thakur A, Kar A, Gerdes H, Venkatesan T (2018) Cyclic vomiting syndrome: epidemiology, diagnosis, and treatment. Clin Auton Res. https://doi.org/10.1007/s10286-0180506-2 (in press)

2. Boronat AC, Ferreira-Maia AP, Matijasevich A, Wang YP (2017) Epidemiology of functional gastrointestinal disorders in children and adolescents: a systematic review. World J Gastroenterol 23:3915-3927

3. Hasler WL (2013) Pathology of emesis: its autonomic basis. Handbook of clinical neurology/edited by PJ Vinken and GW Bruyn 117:337-352

4. Markham C, Diamond SG, Treciokas LJ (1974) Carbidopa in Parkinson disease and in nausea and vomiting of levodopa. Arch Neurol 31:128-133

5. Norcliffe-Kaufmann L, Martinez J, Axelrod F, Kaufmann H (2013) Hyperdopaminergic crises in familial dysautonomia: a randomized trial of carbidopa. Neurology 80:1611-1617

6. Palma JA, Norcliffe-Kaufmann L, Fuente-Mora C, Percival L, Mendoza-Santiesteban C, Kaufmann H (2014) Current treatments in familial dysautonomia. Expert Opin Pharmacother 15:2653-2671

7. Sawai S, Sakakibara R, Kanai K, Kawaguchi N, Uchiyama T, Yamamoto T, Ito T, Liu Z, Hattori T (2006) Isolated vomiting due to a unilateral dorsal vagal complex lesion. Eur Neurol 56:246-248

8. Sierra S, Luquin N, Navarro-Otano J (2018) The endocannabinoid system in cardiovascular function: novel insights and clinical implications. Clin Auton Res 28:35-52 